Pacific Journal of Mathematics

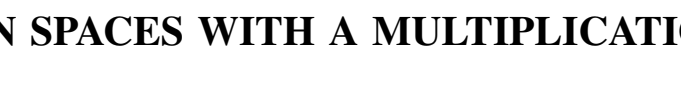




\section{ON SPACES WITH A MULTIPLICATION}

I. M. JAMES

Introduction. This paper is divided into three parts, together with an appendix.

In the first part we discuss the homotopy theory of mappings into a space with a multiplication, such as a topological group. These spaces are more general than the group-like spaces considered by G. W. Whitehead in [6], and our treatment, as far as it goes, is quite different from his. In the second and third parts we apply the theory to the reduced product spaces of [2] and the loop-spaces of [4]. We arrive at useful new definitions of the Hopf construction and the Whitehead product, such that the relations between them are plainly exhibited. In many respects this completes the theory of the suspension triad as developed in [3].

\section{PART I}

\section{Homotopy Theory of a Space with a Multiplication}

1. Preliminary notions. Let $S^{r}$ denote a topological $r$-sphere, with basepoint $e$, where $r \geqq 1$. Let $Z$ be a space with a basepoint, and let $h: S^{p} \times S^{q} \rightarrow Z$ be a map, where $p, q \geqq 1$. By the sections of $h$ we means the maps $f: S^{n} \rightarrow Z, g: S^{a} \rightarrow Z$ which are defined by

$$
f(x)=h(x, e), \quad g(y)=h(e, y) \quad x \in S^{p}, \quad y \in S^{q} .
$$

If $h^{\prime}: S^{p} \times S^{q} \rightarrow Z$ is another map with the same sections as $h$, then the two maps agree on the set of axes

$$
\Sigma=S^{p} \times e \bigvee e \times S^{q},
$$

and since the complement of $\Sigma$ in $S^{p} \times S^{q}$ is an open $(p+q)$-cell the separation element $d\left(h, h^{\prime}\right) \in \pi_{p+q}(Z)$ is defined, as in $\S 10$ below. Of course

$$
d(h, h)=0
$$

In particular, let $Z$ be a space with a multiplication; that is to say, there is a continuous product $x \cdot y \in Z$, where $x, y \in Z$, such that $x \cdot z^{0}=x$ and $z^{0} \cdot y=y$, where $z^{0}$ is the basepoint in $Z$. Let $h$ be as before, and

Received May, 22, 1956.

1 When we consider a map, or homotopy, of one space into another it is always assumed that the image of the basepoint in the one is the basepoint in the other. 
let $h^{\prime}$ be defined by

$$
h^{\prime}(x, y)=f(x) \cdot g(y) \quad x \in S^{p}, \quad y \in S^{q},
$$

where $f, g$ are the sections of $h$. Then $h^{\prime}$ has the same sections as $h$, and we define

$$
\delta(h)=d\left(h^{\prime}, h\right) \in \pi_{p+q}(Z) .
$$

Notice that if $k: S^{p} \times S^{q} \rightarrow Z$ is another map with the same sections then $k^{\prime}=h^{\prime}$ and so

$$
\delta(k)=\delta(h)+d(h, k),
$$

by the addition formula for separation elements ((10.4) below).

Let $w: Z \rightarrow Z^{\prime}$ be a map, where $Z^{\prime}$ is a space with a multiplication. We say that $w$ is multiplicative if

$$
w(x \cdot y)=w(x) \cdot w(y) \quad x, y \in Z .
$$

In that case we have (cf. (10.8))

$$
\delta(w h)=w_{*} \delta(h),
$$

where $w_{*}: \pi_{p+q}(Z) \rightarrow \pi_{p+q}\left(Z^{\prime}\right)$ denotes the homomorphism which is induced by $w$.

2. The pairing of $\pi_{p}(Z)$ with $\pi_{q}(Z)$ to $\pi_{p+q}(Z)$. Let $Z$ be a space with a multiplication, and let $p, q \geqq 1$. With each pair of elements $\alpha \in \pi_{p}(Z)$, $\beta \in \pi_{q}(Z)$ we associate an element $\langle\alpha, \beta\rangle \in \pi_{p+q}(Z)$, as follows. Let $f: S^{p} \rightarrow Z, g: S^{q} \rightarrow Z$ be maps which represent $\alpha, \beta$, respectively. Let $h$, $k: S^{p} \times S^{q} \rightarrow Z$ be the maps which are defined by

$$
h(x, y)=f(x) \cdot g(y), \quad k(x, y)=g(y) \cdot f(x),
$$

where $x \in S^{p}, y \in S^{q}$. Then $h$ and $k$ have the same sections, and we write

$$
\langle\alpha, \beta\rangle=d(h, k) \text {. }
$$

We have at once (cf. (10.8))

TheOREM (2.2). Let $\alpha \in \pi_{p}(Z), \beta \in \pi_{q}(Z)$. Let $w: Z \rightarrow Z^{\prime}$ be a multiplicative map. Then

$$
w_{*}\langle\alpha, \beta\rangle=\left\langle w_{*}(\alpha), w_{*}(\beta)\right\rangle,
$$

where $w_{*}: \pi_{r}(Z) \rightarrow \pi_{r}\left(Z^{\prime}\right)$ denotes the homomorphism induced by $w$.

The type of a map $h: S^{p} \times S^{q} \rightarrow Z$ is the pair of elements $(\alpha, \beta)$, where $\alpha \in \pi_{p}(Z), \beta \in \pi_{q}(Z)$ are the homotopy classes of the sections of 
h. We prove

THEOREM (2.3). Let $Z$ be a space with a multiplication, and let

$$
S^{p} \times S^{q} \stackrel{h}{\rightarrow} Z \stackrel{k}{\leftarrow} S^{q} \times S^{p}
$$

be a pair of maps such that $h(x, y)=k(y, x)$, where $x \in S^{p}, y \in S^{q}$. Let $(\alpha, \beta)$ be the type of $h$, where $\alpha \in \pi_{p}(Z), \beta \in \pi_{q}(Z)$. Then

$$
\langle\alpha, \beta\rangle=\delta(h)-(-1)^{p q} \delta(k) \text {. }
$$

Proof. Let $f: S^{n} \rightarrow Z, g: S^{q} \rightarrow Z$ be the sections of $h$, and let

$$
S^{p} \times S^{q} \stackrel{h^{\prime}}{\rightarrow} \underset{k^{\prime}}{\leftarrow} S^{q} \times S^{p}
$$

be the maps which are defined by

$$
h^{\prime}(x, y)=f(x) \cdot g(y), \quad k^{\prime}(y, x)=g(y) \cdot f(x),
$$

where $x \in S^{p}, y \in S^{q}$. Then

$$
\delta(h)=d\left(h^{\prime}, h\right), \quad \delta(k)=d\left(k^{\prime}, k\right),
$$

by definition. Let $v: S^{n} \times S^{q} \rightarrow S^{q} \times S^{p}$ be the map which interchanges the factors. Then $d\left(k^{\prime} v, k v\right)=(-1)^{k q} d\left(k^{\prime}, k\right)$, by (10.9), since $v$ has degree $(-1)^{p q}$. Therefore

$$
\delta(h)-(-1)^{p q} \delta(k)=d\left(h^{\prime}, h\right)-d\left(k^{\prime} v, k v\right)=d\left(h^{\prime}, k^{\prime} v\right),
$$

by the addition formula for separation elements, since $h=k v$. However

$$
k^{\prime} v(x, y)=k^{\prime}(y, x)=g(y) \cdot f(x),
$$

if $x \in S^{p}$ and $y \in S^{q}$. Hence $d\left(h^{\prime}, k^{\prime} v\right)=\langle\alpha$, $\beta\rangle$, by (2.1), since $\alpha, \beta$ are the homotopy classes of $f, g$, respectively. Therefore

$$
\delta(h)-(-1)^{p q} \delta(k)=\langle\alpha, \beta\rangle,
$$

which proves (2.3).

If we interchange $h$ and $k$ in (2.3), we obtain that

$$
\delta(k)-(-1)^{p q} \delta(h)=\langle\beta, \alpha\rangle,
$$

since $k$ is of type $(\beta, \alpha)$. Hence, and since there exist maps of any given type, we obtain

Corollary (2.4). Let $\alpha \in \pi_{p}(Z), \beta \in \pi_{q}(Z)$. Then

$$
\langle\alpha, \beta\rangle=(-1)^{p q+1}\langle\beta, \alpha\rangle \text {. }
$$


In the next section we shall prove that $\langle\alpha, \beta\rangle$ determines a bilinear pairing of $\pi_{p}(Z)$ with $\pi_{q}(Z)$ to $\pi_{p+q}(Z)$.

3. Products of maps. The proof of the following proposition is omitted, since it is the same as in the case of topological groups (see (16.9) of [5]).

THEOREM (3.1). A space with a multiplication has a commutative fundamental group.

Let $Y$ be a space and let $Z$ be a space with a multiplication. The product of two maps $u, v: Y \rightarrow Z$ is the map $u \cdot v: Y \rightarrow Z$ which is defined by

$$
(u \cdot v)(y)=u(y) \cdot v(y) \quad y \in Y .
$$

In view of (3.1), we write $\pi_{r}(Z)$ additively even when $r=1$. The proof of the following proposition also is the same as in the case of topological groups (see (16.7) of [5]).

Theorem (3.2). Let $v, u: S^{r} \rightarrow Z$ be maps, where $r \geq 1$ and $Z$ is a space with a multiplication. Then the homotopy class of $u \cdot v$ is equal to the sum, in $\pi_{r}(Z)$, of the homotopy classes of $u$ and $v$.

The following lemma is an immediate consequence of (3.2) and the definition of separation elements.

Lemma (3.3). Let $h, k, h^{\prime}, k^{\prime}$ be four maps of $S^{\prime \prime} \times S^{q}$ into $Z$ such that $h$ and $k$ have the same sections, and $h^{\prime}$ and $k^{\prime}$ have the same sections. Then $h \cdot h$ and $k \cdot k^{\prime}$ have the same sections, and their separation element is given by

$$
d\left(h \cdot h^{\prime}, k \cdot k^{\prime}\right)=d(h, k)+d\left(h^{\prime}, k^{\prime}\right) .
$$

We use (3.3) to prove

Theorem (3.4). Let $Z$ be a space with a multiplication. Let $h, h^{\prime}$ be maps of $S^{\prime \prime} \times S^{q}$ into $Z$ of type $(\alpha, \beta),\left(\alpha^{\prime}, \beta^{\prime}\right)$, respectively, where $\alpha, \alpha^{\prime} \in \pi_{p}(Z)$ and $\beta, \beta^{\prime} \in \pi_{q}(Z)$. Then

$$
\delta\left(h \cdot h^{\prime}\right)=\delta(h)+\delta\left(h^{\prime}\right)+\left\langle\alpha^{\prime}, \beta\right\rangle .
$$

The relation we have to prove is invariant under homotopies of $h$ and $h^{\prime}$. Hence there is no real loss of generality if we assume that $h$ and $h^{\prime}$ are such as to satisfy the following condition. Let $(f, g),\left(f^{\prime}, g^{\prime}\right)$ be the sections of $h, h^{\prime}$, respectively, so that $f$ and $f^{\prime}$ are maps of $S^{p}$, and $g$ and $g^{\prime}$ are maps of $S^{q}$. We assume that $f$ is constant over one hemisphere of $S^{\prime \prime}$ and that $f^{\prime}$ is constant over the other; similarly that 
$g$ is constant over one hemisphere of $S^{q}$ and that $g^{\prime}$ is constant over the other. Then, if $x \in S^{p}$ and $y \in S^{q}$, the expressions

$$
f(x) \cdot f^{\prime}(x) \cdot g(y) \cdot g^{\prime}(y), \quad f(x) \cdot g(y) \cdot f^{\prime}(x) \cdot g^{\prime}(y)
$$

do not depend on the order in which the products are taken; it is as though the multiplication on $Z$ were associative. This is expressed more concisely as follows. Let

$$
S^{p} \stackrel{u}{\leftarrow} S^{p} \times S^{q} \stackrel{v}{\rightarrow} S^{q}
$$

denote the canonical projections, and define four maps $F, F^{\prime}, G, G^{\prime}$ of $S^{p} \times S^{q}$ into $Z$ by

$$
F=f u, \quad F^{\prime}=f^{\prime} u ; \quad G=g v, \quad G^{\prime}=g^{\prime} v .
$$

Then the product maps $F \cdot F^{\prime} \cdot G \cdot G^{\prime}$ and $F \cdot G \cdot F^{\prime} \cdot G^{\prime}$ are well-defined.

After these preliminaries, we proceed to prove (3.4). Let $k=F \cdot G$, so that $\delta(h)=d(k, h)$, and let $k^{\prime}=F^{\prime} \cdot G^{\prime}$, so that $\delta\left(h^{\prime}\right)=d\left(k^{\prime}, h^{\prime}\right)$. Then

$$
d\left(k \cdot k^{\prime}, h \cdot h^{\prime}\right)=\delta(h)+\delta\left(h^{\prime}\right),
$$

by (3.3). Let $H=\left(F \cdot F^{\prime}\right) \cdot\left(G \cdot G^{\prime}\right)$. Then $\delta\left(h \cdot h^{\prime}\right)=d\left(H, h \cdot h^{\prime}\right)$, by definition, and hence

$$
\delta\left(h \cdot h^{\prime}\right)=d\left(H, k \cdot k^{\prime}\right)+d\left(k \cdot k^{\prime}, h \cdot h^{\prime}\right),
$$

by the addition formula for separation elements. Hence

$$
\delta\left(h \cdot h^{\prime}\right)=\delta(h)+\delta\left(h^{\prime}\right)+d\left(H, k \cdot k^{\prime}\right),
$$

by (3.5). However,

$$
\begin{aligned}
\left\langle\alpha^{\prime}, \beta\right\rangle & =d\left(F^{\prime \prime} \cdot G, G \cdot F^{\prime}\right), \text { by definition, } \\
& =d(F, F)+d\left(F^{\prime} \cdot G, G \cdot F^{\prime}\right)+d\left(G^{\prime}, G^{\prime}\right), \text { by }(1.1), \\
& =d\left(F \cdot F^{\prime} \cdot G, F \cdot G \cdot F^{\prime}\right)+d\left(G^{\prime}, G^{\prime}\right), \text { by }(3.3), \\
& =d\left(F \cdot F^{\prime} \cdot G \cdot G^{\prime}, F \cdot G \cdot F^{\prime} \cdot G^{\prime}\right), \text { by }(3.3), \\
& =d\left(H, k \cdot k^{\prime}\right),
\end{aligned}
$$

by definition. Hence it follows from (3.6) that

$$
\delta\left(h \cdot h^{\prime}\right)=\delta(h)+\delta\left(h^{\prime}\right)+\left\langle\alpha^{\prime}, \beta\right\rangle,
$$

which proves (3.4).

As an application of (3.4) we prove".

Theorem (3.7). Let $Z$ be a space with a multiplication, and let $p, q \geqq 1$. Then the transformation $(\alpha, \beta) \rightarrow\langle\alpha, \beta\rangle$ determines a bilinear

2 This can also be proved directly. 
pairing of $\pi_{p}(Z)$ with $\pi_{q}(Z)$ to $\pi_{p+q}(Z)$.

We first show that

$$
\left\langle\alpha+\alpha^{\prime}, \beta\right\rangle=\langle\alpha, \beta\rangle+\left\langle\alpha^{\prime}, \beta\right\rangle,
$$

where $\alpha, \alpha^{\prime} \in \pi_{p}(Z), \beta \in \pi_{q}(Z)$. For let $f, f^{\prime}: S^{p} \rightarrow Z$ be maps which represent $\alpha, \alpha^{\prime}$, respectively, such that $f$ is constant over one hemi-sphere of $S^{p}$ and $f^{\prime}$ is constant over the other. Let $g: S^{q} \rightarrow Z$ represent $\beta$, and let $h, h^{\prime}$ be the maps of $S^{p} \times S^{a}$ into $Z$ which are defined by

$$
h(x, y)=g(y) \cdot f(x), \quad h^{\prime}(x, y)=f^{\prime}(x),
$$

where $x \in S^{p}, y \in S^{q}$. Then

$$
\left(h \cdot h^{\prime}\right)(x, y)=(g(y) \cdot f(x)) \cdot f^{\prime}(x)=g(y) \cdot\left(f(x) \cdot f^{\prime}(x)\right),
$$

and so $\delta\left(h \cdot h^{\prime}\right)=\langle\gamma, \beta\rangle$, by (2.1), where $\gamma$ denotes the homotopy class of $f \cdot f^{\prime}$. But $\gamma=\alpha+\alpha^{\prime}$, by (3.2), and so

$$
\begin{aligned}
\left\langle\alpha+\alpha^{\prime}, \beta\right\rangle & =\delta\left(h \cdot h^{\prime}\right) \\
& =\delta(h)+\delta\left(h^{\prime}\right)+\left\langle\alpha^{\prime}, \beta\right\rangle, \text { by }(3.4), \\
& =\langle\alpha, \beta\rangle+\left\langle\alpha^{\prime}, \beta\right\rangle,
\end{aligned}
$$

since $\delta\left(h^{\prime}\right)=d\left(h^{\prime}, h^{\prime}\right)=0$, by (1.1). This proves (3.8). Linearity on the right follows from (3.8) and (2.4). Hence the proof of (3.7) is complete.

\section{PART II}

\section{Application to Reduced Product Complexes}

4. The reduced product complex. Throughout this part of the paper, $A$ will denote a countable $C W$-complex with precisely one 0 -cell, say $a^{0}$. Let $A_{\infty}$ denote the reduced product complex of $A$, as defined in [2]. We recall that $A_{\infty}$ is a countable $C W$-complex which contains $A$ as a subcomplex, and that $A_{\infty}$ carries an associative multiplication with $a^{0}$ as unit element. Let $I$ denote the interval $0 \leqq t \leqq 1$. Let $\hat{A}$ denote the suspension of $A$, that is the space which is obtained from the topological product $A \times I$ by identifying $A \times \dot{I} \cup a^{0} \times I$ to a point. The points of $\hat{A}$ are represented by pairs $(a, t)$, where $a \in A$ and $t \in I$, with the identification being tacitly understood. We also identify each point $a \in A$ with $\left(a, \frac{1}{2}\right) \in \hat{A}$, so that $A$ is embedded in $\hat{A}$. The suspension triad of $A$ is the triad

$$
\left(\hat{A} ; C_{+}, C_{-}\right),
$$

in which $C_{-}, C_{+}$are the half-cones where $t \leqq \frac{1}{2}, t \geqq \frac{1}{2}$, respectively, so 
that

$$
\hat{A}=C_{+} \cup C_{-}, \quad A=C_{+} \cap C_{-} .
$$

The relation between the reduced product complex and the suspension triad is expressed in the following commutative diagram, where $\phi$ denotes the canonical isomorphism which is defined in $\S 10$ of [3].

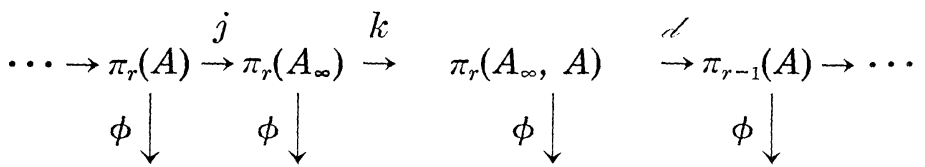

$$
\begin{aligned}
& \cdots \rightarrow \pi_{r}(A) \underset{E}{\rightarrow} \pi_{r+1}(\hat{A}) \underset{i}{\rightarrow} \pi_{r+1}\left(\hat{A} ; C_{+}, C_{-}\right) \underset{\Delta}{\rightarrow} \pi_{r-1}(A) \rightarrow \cdots \text {. }
\end{aligned}
$$

The top line of the diagram is part of the homotopy sequence of the pair $\left(A_{\infty}, A\right)$, so that $j, k$ are injections, and $\alpha$ is the boundary operator. The bottom line is part of the suspension sequence of $A$, as defined in [3], so that $E$ is the suspension operator, $i$ is the injection, and $\Delta$ is the repeated boundary operator. We recall from [3] that $\phi$ maps $\pi_{r}(A)$ identically, so that the commutativity of (4.1) is expressed by the following relations (cf. (10.2) of [3]).

$$
\begin{aligned}
& \text { (a) }\{j=E \text {, } \\
& \text { (b) }\{\phi=\phi k \text {, } \\
& \text { (c) } \Delta \phi=d \text {. }
\end{aligned}
$$

Let $B$ a countable $C W$-complex with precisely one 0 -cell, say $b^{0}$, and let $f: A \rightarrow B$ be a map such that $f\left(a^{0}\right)=b^{0}$. Then the induced mapping $f_{\infty}: A_{\infty} \rightarrow B_{\infty}$, as defined in $\S 1$ of [2], is multiplicative in the sense of $\S 1$. Let $\hat{f}: \hat{A} \rightarrow \hat{B}$ denote the suspension of $f$, which is defined by

$$
\hat{f}(a, t)=(f(a), t) \quad a \in A, \quad t \in I .
$$

Then $\hat{f}$ maps the suspension triad of $A$ into the suspension triad of $B$, and hence induces a homomorphism of the suspension sequence of $A$ into the suspension sequence of $B$. We denote this homomorphism by $f_{*}$, and we also denote by $f_{*}$ the homomorphism of the homotopy sequence of $\left(A_{\infty}, A\right)$ into the homotopy sequence of $\left(B_{\infty}, B\right)$ which is induced by $f_{\infty}$. By (10.5) of [3] these homomorphisms are related by

$$
\phi f_{*}=f_{*} \phi .
$$

5. The Hopf construction. Let $A$ mean the same as in $\S 4$, and let $p, q \geqq 1$. A pairing of $\pi_{p}(A)$ with $\pi_{q}(A)$ to $\pi_{p+q}\left(A_{\infty}, A\right)$ is defined as follows. Let $\gamma$ denote the positive generator of the infinite cyclic group 
$\pi_{p+q}\left(S^{p} \times S^{q}, \Sigma\right)$, where $\Sigma=S^{p} \times e \bigcup e \times S^{q}$ and the orientations are the same as in [3]. Let $f, g$ be maps of $S^{p}, S^{q}$ into $A$ which represent $\alpha \in \pi_{p}(A)$, $\beta \in \pi_{q}(A)$, respectively. Let $h:\left(S^{p} \times S^{q}, \Sigma\right) \rightarrow\left(A_{\infty}, A\right)$ denote the map which is defined by

$$
h(x, y)=f(x) \cdot g(y) \quad x \in S^{p}, \quad y \in S^{q} .
$$

Then we define $\alpha \times \beta=h_{*}(\gamma)$, where

$$
h_{*}: \pi_{p+q}\left(S^{p} \times S^{q}, \Sigma\right) \rightarrow \pi_{p+q}\left(A_{\infty}, A\right)
$$

denotes the homomorphism induced by $h$. We write

$$
\{\alpha, \beta\}=\phi(\alpha \times \beta) \in \pi_{p+q+1}\left(\hat{A} ; C_{+}, C_{-}\right),
$$

and we refer to $\{\alpha, \beta\}$ as the triad Whitehead product of $\alpha$ and $\beta$, in accordance with (7.1) of [3].

Let us apply the theory of Part I to the space with multiplication $A_{\infty}$. By taking representatives we obtain from (2.1) that

$$
k\langle j(\alpha), j(\beta)\rangle=\alpha \times \beta-(-1)^{p q} \beta \times \alpha,
$$

and hence, by (4.2b) and (5.1), we have

$$
i \phi\langle j(\alpha), j(\beta)\rangle=\{\alpha, \beta\}-(-1)^{p q}\{\beta, \alpha\} .
$$

Now suppose that there exists a map $h: S^{p} \times S^{q} \rightarrow A$ of type $(\alpha, \beta)$. Let $h^{\prime}$ denote the inclusion of $h$ into $A_{\infty}$. By taking representatives we obtain at once that $k \delta\left(h^{\prime}\right)=\alpha \times \beta$, and so we conclude from (4.2b) and (5.1) that

$$
i \phi \delta\left(h^{\prime}\right)=\{\alpha, \beta\} .
$$

Recall that the Hopf construction, as defined in [3], assigns an element $c(h) \in \pi_{p+q+1}(\hat{A})$ to each map $h: S^{p} \times S^{q} \rightarrow A$, and is characterized uniquely by the following three properties. First, let $h$ have type $(\alpha, \beta)$, where $\alpha \in \pi_{p}(A), \beta \in \pi_{q}(A)$. Then

$$
i c(h)=\{\alpha, \beta\} .
$$

Secondly, let $B$ mean the same as in $\S 4$, and let $f: A \rightarrow B$ be a map such that $f\left(a^{0}\right)=b^{0}$. Then

$$
c(f h)=f_{*} c(h) .
$$

Thirdly, let $A=S^{p} \times S^{q}$, and let $h$ be either of the projections

$$
(x, y) \rightarrow(x, e), \quad(x, y) \rightarrow(e, y),
$$

where $x \in S^{p}, y \in S^{q}$. Then 


$$
c(h)=0 \text {. }
$$

The uniqueness of this characterization follows from (8.2), (8.3) and (8.4) of [3]. We use it to prove

Theorem (5.7). Let $h: S^{p} \times S^{q} \rightarrow A$ be a map, and let $h^{\prime}$ denote its inclusion into $A_{\infty}$. Let $c(h)$ denote the element of $\pi_{p+q+1}(\hat{A})$ which is obtained from $h$ by the Hopf construction, and let $\delta\left(h^{\prime}\right)$ denote the element of $\pi_{p+q}\left(A_{\infty}\right)$ which is obtained from $h^{\prime}$ as in $\S 1$. Then $c(h)=\phi \delta\left(h^{\prime}\right)$.

Let $\gamma(h)=\phi \delta\left(h^{\prime}\right)$. We check that $\gamma(h)$ satisfies (5.4), (5.5) and (5.6) as well as $c(h)$. For (5.4) follows from (5.3), in the case of $\gamma(h)$, and (5.6) follows from (1.1). Consider (5.5), where we have a map $f: A \rightarrow B$. Let $f_{\infty}: A_{\infty} \rightarrow B_{\infty}$ denote the multiplicative map which $f$ determines. Then $f_{\infty} h^{\prime}$ is equal to the inclusion of $f h$ into $B_{\infty}$, and so

$$
\begin{aligned}
\gamma(f h)=\phi \delta\left(f_{\infty} h^{\prime}\right) & =\phi f_{*} \delta\left(h^{\prime}\right), \text { by }(1.4), \\
& =f_{*} \phi \delta\left(h^{\prime}\right), \text { by }(4.3), \\
& =f_{*} \gamma(h), \text { by definition } .
\end{aligned}
$$

Therefore $\gamma(h)$ satisfies all three conditions, whence $\gamma(h)=c(h)$. This proves (5.7).

6. The Whitehead product. Let $X$ be a space with a basepoint, and let $p, q \geqq 1$. The Whitehead product of a pair of elements $(\xi, \eta)$, where $\xi \in \pi_{p+1}(X), \eta \in \pi_{q+1}(X)$, is an element of $\pi_{p+q+1}(X)$, and is denoted by $[\xi, \eta]$. In $\S 9$ we shall prove a general theorem about this product which implies the following in case $X$ is the suspension of $A$, where $A$ is a conplex as in $\S 4$.

Theorem (6.1). Let $\lambda \in \pi_{p}\left(A_{\infty}\right), \mu \in \pi_{q}\left(A_{\infty}\right)$. Then

$$
\phi\langle\lambda, \mu\rangle=(-1)^{p}[\phi(\lambda), \phi(\mu)] \text {. }
$$

Since $\phi j=E$, by (4.2a), we have the following three corollaries in case $\lambda=j(\alpha)$ or $\mu=j(\beta)$, where $\alpha \in \pi_{p}(A), \beta \in \pi_{q}(A)$.

CoRollary (6.2). Let $\lambda \in \pi_{p}\left(A_{\infty}\right), \beta \in \pi_{q}(A)$. Then

$$
\phi\langle\lambda, j(\beta)\rangle=(-1)^{p}[\phi(\lambda), E(\beta)] .
$$

Corollary (6.3). Let $\alpha \in \pi_{p}(A), \mu \in \pi_{q}\left(A_{\infty}\right)$. Then

$$
\phi\langle j(\alpha), \mu\rangle=(-1)^{p}[E(\alpha), \phi(\mu)] .
$$

Corollary (6.4). Let $\alpha \in \pi_{p}(A), \beta \in \pi_{q}(A)$. Then 


$$
\phi\langle j(\alpha), j(\beta)\rangle=(-1)^{p}[E(\alpha), E(\beta)] .
$$

Hence and from (5.2) we obtain the first commutation law for triad Whitehead products (cf. (2.4) of [3]):

Corollary (6.5). Let $\alpha \in \pi_{p}(A), \beta \in \pi_{q}(A)$. Then

$$
\{\alpha, \beta\}-(-1)^{p q}\{\beta, \alpha\}=(-1)^{p} i[E(\alpha), E(\beta)] \text {. }
$$

As defined in [2], $A_{\infty}$ is filtered by a sequence of subcomplexes $A_{0} \subset A_{1} \subset \cdots \subset A_{m} \subset \cdots$, where $A_{0}=a^{0}$ and $A_{1}=A$. The reduced product filtration of $\pi_{r+1}(\hat{A})$ is defined as follows (cf. $\S 13$ of [3]). We say that an element $\gamma \in \pi_{r}\left(A_{\infty}\right)$ has filtration $m(m \geq 1)$ if $\gamma$ can be represented by a map of $S^{r}$ whose image is contained in $A_{m}$ but not by one whose image is contained in $A_{m-1}$. We also say that the zero element has filtration zero. The reduced product filtration of $\pi_{r+1}(\hat{A})$ is obtained from this by applying the canonical isomorphism $\phi$. We supplement the results of [3] by

CoRollary (6.6). Let $\xi \in \pi_{p+1}(\hat{A}), \eta \in \pi_{q+1}(\hat{A})$ be elements with filtrations $m, n$, respectively. Then the filtration of the Whitehead product $[\xi, \eta] \in \pi_{p+q+1}(\hat{A})$ does not exceed $m+n$.

This follows from (6.1). For let $\alpha \in \pi_{p}\left(A_{\infty}\right), \beta \in \pi_{q}\left(A_{\infty}\right)$ be elements such that $\varphi(\alpha)=\xi, \varphi(\beta)=\eta$. By hypothesis, there exist maps $f: S^{p} \rightarrow A_{\infty}$, $S^{q} \rightarrow A_{\infty}$, representing $\alpha, \beta$, respectively, such that $f S^{p} \subset A_{m}, g S^{q} \subset A_{n}$. Now $A_{m} \cdot A_{n}=A_{n} \cdot A_{m}=A_{m+n}$, by the definition of $A_{\infty}$. Hence $h\left(S^{p} \times S^{q}\right) \subset$ $A_{m+n}$, where $h$ denotes either of the maps

$$
f(x) \cdot g(y) \leftarrow(x, y) \rightarrow g(y) \cdot f(x) \quad x \in S^{p}, \quad y \in S^{q} .
$$

Therefore $\langle\alpha, \beta\rangle$ can be represented by a map of $S^{p+q}$ into $A_{m+n}$, so that the filtration of $\langle\alpha, \beta\rangle$, and hence of $\phi\langle\alpha, \beta\rangle$, does not exceed $m+n$. Hence, by (6.1), the filtration of $[\phi(\alpha), \phi(\beta)]$ does not exceed $m+n$, which proves $(6.6)$.

\section{PART III}

\section{Application to LOOP-SpaCeS}

7. The loop-space (in the sense of Moore). Let $X$ be a space with basepoint $x_{0}$. By a loop in $X$ we mean a pair $(f, s)$, where $s \geqq 0$ and $f$ is a map of the interval $0 \leqq t \leqq s$ into $X$ such that $f(0)=f(s)=x_{0}$. The composition of $(f, s)$ with another loop $\left(f^{\prime}, s^{\prime}\right)$ is the loop $\left(f^{\prime \prime}, s+s^{\prime}\right)$, where 


$$
f^{\prime \prime}(t)= \begin{cases}f(t) & (0 \leqq t \leqq s), \\ f^{\prime}(t-s) & \left(s \leqq t \leqq s+s^{\prime}\right) .\end{cases}
$$

Let $A$ denote the set of loops with the topology defined in $\S 2$ of [4]; we call $A$ the loop-space of $X$. The ordinary space of loops, $\Omega$, consists of those loops $(f, s)$ such that $s=1$. Let $x^{0}$ denote the loop $(f, 0)$, where $f(0)=x_{0}$. The product in $A$ which is defined by composition of loops is associative, and admits $x^{0}$ as a unit element. In $\S 2$ of [4] Moore asserts the following propositions. We omit the proofs, which are straightforward but tedious.

Theorem (7.1). (a) The product in $\Lambda$ is continuous; and (b) $\Omega$ is a deformation retract of $\Lambda$.

Let us represent $S^{r+1}$ as the suspension of $S^{r}$, as in $\S 1$ of [3], so that $(x, t) \in S^{r+1}$ if $x \in S^{r}$ and $t \in I$. Let $h: S^{r} \rightarrow \Lambda$ be a map, and let $h(x)=$ $(f, s)$, say, where $s \geqq 0$ and $f$ maps the interval $0 \leqq t \leqq s$ into $X$. Then a map $h^{\prime}: S^{r+1} \rightarrow X$ is defined by $h^{\prime}(x, t)=f(s t)$, where $0 \leqq t \leqq 1$. The transformation $h \rightarrow h^{\prime}$ is invariant under homotopy, and therefore it defines a function $\psi: \pi_{r}(\Lambda) \rightarrow \pi_{r+1}(X)$. We prove

\section{THEOREM (7.2). The funcion $\psi$ is an isomorphism (onto).}

For let $i_{*}$ denote the injection of $\pi_{r}(\Omega)$ into $\pi_{r}(\Lambda)$, which is an isomorphism by (7.1b). By taking representatives we find that $\psi i_{*}=\theta$, the Hurewicz isomorphism of $\pi_{r}(\Omega)$ onto $\pi_{r+1}(X)$. Hence $\psi$ is an isomorphism, which proves (7.2). Notice also that $\psi$ is natural. To be precise, let $X^{\prime}$ be a space with a basepoint and let $h: X \rightarrow X^{\prime}$ be a map. If $(f, s)$ is a loop in $X$, where $s \geqq 0$ and $f$ maps the interval $0 \leqq t \leqq s$ into $X$, then $(h f, s)$ is a loop in $X^{\prime}$. Let $\bar{h}: A \rightarrow \Lambda^{\prime}$ denote the multiplicative map which is defined by $\bar{h}(f, s)=(h f, s)$, where $\Lambda^{\prime}$ is the loop-space of $X^{\prime}$. Then by taking representatives it follows at once that

$$
\psi^{\prime} \bar{h}_{*}=h_{*} \psi,
$$

where $\psi^{\prime}$ means the same for $X^{\prime}$ as $\psi$ does for $X$, and where $h_{*}, \bar{h}_{*}$ are the homomorphisms induced by $h, \bar{h}$, as shown in the following diagram:

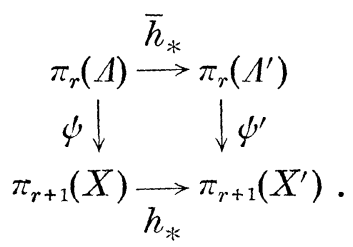

8. The canonical isomorphism. Let $A$ be a space, with basepoint 
$a^{0}$, on which a real-valued continuous function $d$ is defined which is positive except that $d\left(a^{0}\right)=0$. Let $\hat{A}$ denote the suspension of $A$, and let $\Lambda$ denote the loop-space of $\hat{A}$. Let $x^{0} \in \Lambda$ denote the trivial loop at the suspension of $a^{0}$. Then a map $u: A \rightarrow \Lambda$ is defined as follows. Let $a \in A$ and let $\alpha=d(a)$. We define $u\left(a^{0}\right)=x^{0}$. Let $a \neq a^{0}$. Then $\alpha>0$ and we define $u(\alpha)=(f, \alpha)$, where $f$ is the map of the interval $0 \leqq t \leqq \alpha$ into $\hat{A}$ which is defined by $f(t)=(a, t / \alpha)$. Of course $u$ depends on $d$, but since the set of functions $d$ is convex it follows that any two maps $u$ are homotopic. The topology of $\Lambda$ is such that $u$ is a homeomorphism into $\Lambda$, so that we regard $A$ as a subspace of $\Lambda$.

We now define a homomorphism, $\psi$, of the homotopy sequence of the pair $(A, A)$ into the suspension sequence of $A$, as shown in the following diagram.

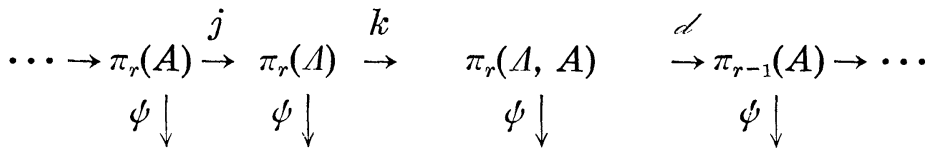

$$
\begin{aligned}
& \cdots \rightarrow \pi_{r}(A) \underset{E}{\rightarrow} \pi_{r+1}(\hat{A}) \underset{i}{\rightarrow} \pi_{r+1}\left(\hat{A} ; C_{+}, C_{-}\right) \underset{\Delta}{\rightarrow} \pi_{r-1}(A) \rightarrow \cdots .
\end{aligned}
$$

We define $\psi$ as follows. Let ${ }^{3}$ denote the convex hull of $S^{r-1}$, so that points of $V^{r}$ are represented by pairs $(s, x)$, where $x \in S^{r-1}$ and $0 \leqq s \leqq 1$, such that $(0, x)=e,(1, x)=x$. Let $V^{r+1}$ denote the suspension of $V^{r}$, so that points of $V^{r+1}$ are represented by pairs $(y, t)$, where $y \in V^{r}$ and $0 \leqq t \leqq 1$. Let $h: V^{r} \rightarrow \Lambda$ be a map, and let $h(y)=(f, s)$, say, where $s \geqq 0$ and $f$ maps the interval $0 \leqq t \leqq s$ into $\hat{A}$. Let $h^{\prime}$ : $V^{r+1} \rightarrow \hat{A}$ be the map defined by $h^{\prime}(y, t)=f(s t)$. Since $h^{\prime} S^{r}=a^{0}$ if $h S^{r-1}=$ $x^{0}$, we define $\psi$ on $\pi_{r}(A)$ to be the homomorphism induced by the transformation $h \rightarrow h^{\prime}$. It is easy to check that $\psi$ means the same here as in (7.2). If $h S^{r-1} \subset A$ then $h^{\prime}$ maps one hemisphere of $S^{r}$ into $C_{+}$and the other into $C_{-}$, so that the transformation $h \rightarrow h^{\prime}$ also induces a homomorphism of $\pi_{r}(\Lambda, A)$ into $\pi_{r+1}\left(\hat{A} ; C_{+}, C_{-}\right)$. Thus we define $\phi$ on $\pi_{r}(\Lambda, A)$, and the definition is completed by setting $\psi$ to be the identity on $\pi_{r}(A)$. It is easily verified that these definitions make (8.1) commutative, that is, that

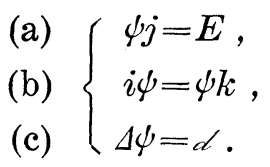

Since $\psi$ maps $\pi_{r}(A)$ identically, by definition, and maps $\pi_{r}(\Lambda)$ isomorphically, by (7.2), we obtain by an application of the five lemma:

\footnotetext{
${ }^{3}$ See $\$ 1$ of $[\mathbf{3}]$ for details of these representations.
} 
THEOREM (8.3). As shown in (8.1), $\psi$ is an isomorphism of the homotopy sequence of pair $(\Lambda, A)$ onto the suspension sequence of $A$.

Now let $A$ be a complex as in $\S 4$, and consider the reduced product complex $A_{\infty}$. We extend the inclusion map $u: A \rightarrow \Lambda$ to a multiplicative map $w: A_{\infty} \rightarrow \Lambda$, as follows. Let $a_{*} \in A_{\infty}$, so that $a_{*}=a_{1} \cdot a_{2} \cdot \cdots \cdot a_{n}$, say, where $a_{1}, a_{2}, \cdots, a_{n} \in A$. Then we define

$$
w\left(a_{*}\right)=u\left(a_{1}\right) \cdot u\left(a_{2}\right) \cdot \cdots \cdot u\left(a_{n}\right) \text {. }
$$

Notice that $w$ is nonsingular, although $A_{\infty}$ is not mapped homeomorphically unless $A=a^{0}$. Let $w_{*}$ denote the homomorphism of the homotopy sequence of $\left(A_{\infty}, A\right)$ into the homotopy sequence of $(A, A)$ which is induced by $w$, and let $\phi$ denote the canonical isomorphism of the homotopy sequence of $\left(A_{\infty}, A\right)$ onto the suspension sequence of $A$, as in (4.1). It follows from the definition of $\phi$ in $\S 10$ of [3] that

$$
\phi=\psi w_{*} .
$$

Hence and from (8.3) we obtain

THEOREM (8.5). The homomorphism $w_{*}$ maps the homotopy sequence of the pair $\left(A_{\infty}, A\right)$ isomorphically onto the homotopy sequence of the pair $(\Lambda, A)$.

Thus $w$ is an algebraic homotopy equivalence of the pair, in the sense of [2]. Let us also denote by $w_{*}$ the homomorphism in singular homology which $w$ induces. Then from (8.5) of [2] we obtain

CoRollaRY (8.6). The homomorphism $w_{*}$ maps the singular homology sequence of the pair $\left(A_{\infty}, A\right)$ isomorphically onto the singular homology sequence of the pair $(\Lambda, A)$.

The next section is devoted to proving:

Theorem (8.7). Let $A$ denote the loop-space of a space $X$, and let $\xi \in \pi_{p}(\Lambda), \eta \in \pi_{q}(\Lambda)$, where $p, q \geqq 1$. Then

$$
\psi\langle\xi, \eta\rangle=(-1)^{p}[\psi(\xi), \psi(\eta)] .
$$

We conclude the present section by showing how (6.1) is deduced from (8.7). Let $A$ be a complex as in $\S 6$, and let $X=\hat{A}$ in (8.7). Then if $\lambda \in \pi_{p}\left(A_{\infty}\right), \mu \in \pi_{q}\left(A_{\infty}\right)$ are the elements given in (6.1) we have that

$$
w_{*}\langle\lambda, \mu\rangle=\left\langle w_{*}(\lambda), w_{*}(\mu)\right\rangle,
$$

by (2.2), since $w$ is multiplicative. Moreover,

$$
\psi\left\langle w_{*}(\lambda), w_{*}(\mu)\right\rangle=(-1)^{p}\left[\psi w_{*}(\lambda), \phi w_{*}(\mu)\right],
$$

by (8.7). Hence and from (8.4) we conclude that 


$$
\begin{aligned}
\varphi\langle\lambda, \mu\rangle=\phi w_{*}\langle\lambda, \mu\rangle & =(-1)^{p}\left[\psi w_{*}(\lambda), \phi w_{*}(\mu)\right] \\
& =(-1)^{p}[\phi(\lambda), \phi(\mu)] .
\end{aligned}
$$

Thus (6.1) follows from (8.7), and it only remains for us to prove (8.7).

9. Proof of (8.7). Let $A$ be a countable $C W$-complex with only one 0-cell, and let $u: A \rightarrow \Lambda$ be the inclusion map, where $\Lambda$ denotes the loop-space of $\hat{A}$. We prove first of all

Theorem (9.1). Let $h: S^{p} \times S^{q} \rightarrow A$ be a map, where $p, q \geqq 1$, and let $c(h)$ denote the element of $\pi_{p+q+1}(\hat{A})$ which is obtained from $h$ by the Hopf construction. Let $\delta(u h)$ denote the element of $\pi_{p+q}(\Lambda)$ which is obtained from the inclusion of $h$ into $A$ as in $\S 1$. Then $c(h)=\psi \delta(u h)$.

Proof. We have $u h=w h^{\prime}$, by the definition of $w$, where $h^{\prime}$ denotes the inclusion of $h$ into $A_{\infty}$. Hence

$$
\delta(u h)=\delta\left(w h^{\prime}\right)=w_{*} \delta\left(h^{\prime}\right),
$$

by (1.4), since $w$ is multiplicative. Hence

$$
\begin{aligned}
\psi \delta(u h)=\psi w_{*} \delta\left(h^{\prime}\right) & =\phi \delta\left(h^{\prime}\right), \text { by }(8.4), \\
& =c(h), \text { by }(5.7) .
\end{aligned}
$$

This proves (9.1). We deduce

Corollary (9.2). Let $h_{1}, h_{2}: S^{p} \times S^{q} \rightarrow A$ be maps which have the same sections, and let $d\left(h_{1}, h_{2}\right)$ denote their separation element in $\pi_{p+q}(A)$. Then

$$
c\left(h_{2}\right)=c\left(h_{1}\right)+E d\left(h_{1}, h_{2}\right)
$$

Proof. We have

$$
\begin{aligned}
\delta\left(u h_{2}\right)-\delta\left(u h_{1}\right) & =d\left(u h_{1}, u h_{2}\right), \text { by }(1.3), \\
& =j d\left(h_{1}, h_{2}\right) .
\end{aligned}
$$

by the naturality of the separation element. Therefore

$$
\psi \delta\left(u h_{2}\right)-\psi \delta\left(u h_{1}\right)=\psi j d\left(h_{1}, h_{2}\right)=E d\left(h_{1}, h_{2}\right),
$$

by (8.2a). Hence (9.2) follows from (9.1).

Now take $A=S^{p} \times S^{q}$ and let $\alpha \in \pi_{p}(A), \beta \in \pi_{q}(A)$ be the homotopy classes of the maps of $S^{p}, S^{q}$ into $A$ which are given by

$$
x \rightarrow(x, e), \quad\left(x \in S^{p}\right) ; \quad y \rightarrow(e, y), \quad\left(y \in S^{q}\right) ;
$$


respectively. We prove that

$$
\psi\langle j(\alpha), j(\beta)\rangle=(-1)^{p}[E(\alpha), E(\beta)] .
$$

For let $h: S^{p} \times S^{q} \rightarrow A$ denote the identity map, and let $v: S^{q} \times S^{p} \rightarrow A$ denote the map which interchanges the factors. Since $u h$ has type $(j(\alpha), j(\beta))$, it follows from $(2.3)$ that

$$
\langle j(\alpha), j(\beta)\rangle=\delta(u h)-(-1)^{p q} \delta(u v) \text {. }
$$

Therefore

$$
\begin{aligned}
\psi\langle j(\alpha), j(\beta)\rangle & =\psi \delta(u h)-(-1)^{p q} \psi \delta(u v) \\
& =c(h)-(-1)^{p q} c(v), \text { by }(9.1), \\
& =(-1)^{p}[E(\alpha), E(\beta)],
\end{aligned}
$$

by (2.19) of [3]. This proves (9.3).

We continue to consider $A=S^{p} \times S^{q}$, and we denote the set of axes $S^{p} \times e \cup e \times S^{q}$ by $A^{\prime}$. Then $\hat{A}$ contains $\hat{A}^{\prime}$, which we identify with $S^{n+1} \times e \cup e \times S^{q+1}$. Let $\Lambda^{\prime}$ denote the loop-space of $\hat{A}^{\prime}$, regarded as a subspace of $A$, and let $j^{\prime}, E^{\prime}$ and $\psi^{\prime}$ mean the same in the case of $A^{\prime}$ as do $j, E$ and $\psi$ in the case of $A$. Thus, if $k_{*}$ denotes any of the homomorphisms induced by the inclusion map $k: A^{\prime} \rightarrow A$ we have the relations
(a) $\quad k_{*} j^{\prime}=j k_{*}$,
(b) $\left\{k_{*} E^{\prime}=E k_{*}\right.$,
(c) $\quad k_{*} \psi^{\prime}=\psi k_{*}$.

Let $\alpha^{\prime} \in \pi_{p}\left(A^{\prime}\right), \beta^{\prime} \in \pi_{q}\left(A^{\prime}\right)$ denote the homotopy classes of the maps of $S^{p}, S^{q}$ into $A^{\prime}$ which are defined by

$$
x \rightarrow(x, e), \quad\left(x \in S^{p}\right) ; \quad y \rightarrow(e, y), \quad\left(y \in S^{q}\right) ;
$$

respectively. Since $\alpha=k_{*}\left(\alpha^{\prime}\right)$ and $\beta=k_{*}\left(\beta^{\prime}\right)$ it follows that

$$
\begin{aligned}
\psi k_{*}\left\langle j^{\prime}\left(\alpha^{\prime}\right), j^{\prime}\left(\beta^{\prime}\right)\right\rangle & =\psi\left\langle k_{*} j^{\prime}\left(\alpha^{\prime}\right), k_{*} j^{\prime}\left(\beta^{\prime}\right)\right\rangle, \text { by }(2.2), \\
& =\psi\langle j(\alpha), j(\beta)\rangle, \text { by }(9.4 \mathrm{a}), \\
& =(-1)^{p}[E(\alpha), E(\beta)], \text { by }(9.3), \\
& =(-1)^{p}\left[k_{*} E^{\prime}\left(\alpha^{\prime}\right), k_{*} E^{\prime}\left(\beta^{\prime}\right)\right], \text { by }(9.4 \mathrm{~b}), \\
& =(-1)^{p} k_{*}\left[E^{\prime}\left(\alpha^{\prime}\right), E^{\prime}\left(\beta^{\prime}\right)\right],
\end{aligned}
$$

by the naturality of the Whitehead product. Hence

$$
k_{*} \psi^{\prime}\left\langle j^{\prime}\left(\alpha^{\prime}\right), j^{\prime}\left(\beta^{\prime}\right)\right\rangle=(-1)^{p} k_{*}\left[E^{\prime}\left(\alpha^{\prime}\right), E^{\prime}\left(\beta^{\prime}\right)\right],
$$


by $(9.4 \mathrm{c})$. But since $\hat{A}^{\prime}$ is a retract of $\hat{A}$, the injection

$$
k_{*}: \pi_{p+q+1}\left(\hat{A}^{\prime}\right) \rightarrow \pi_{p+q+1}(\hat{A})
$$

is an isomorphism into. Therefore we conclude that

$$
\psi^{\prime}\left\langle j^{\prime}\left(\alpha^{\prime}\right), j^{\prime}\left(\beta^{\prime}\right)\right\rangle=(-1)^{p}\left[E^{\prime}\left(\alpha^{\prime}\right), E^{\prime}\left(\beta^{\prime}\right)\right] .
$$

Continue with the same meaning for $A^{\prime}$, $\Lambda^{\prime}$ etc., but now let $\Lambda$ mean the loop-space of $X$, as in (8.7). Let $\xi \in \pi_{p}(\Lambda), \eta \in \pi_{q}(\Lambda)$ be the elements given in (8.7). Let $f, g$ be maps of $S^{p+1}, S^{q+1}$ into $X$ which represent $\psi(\xi), \psi(\eta)$, respectively, and let $h: \hat{A} \rightarrow X$ denote the map which is defined by

$$
h(x, e)=f(x), \quad\left(x \in S^{p+1}\right) ; \quad h(e, y)=g(y), \quad\left(y \in S^{q+1}\right) .
$$

Let $\bar{h}: \Lambda^{\prime} \rightarrow \Lambda$ denote the map defined by composing loops with $h$, as in $\S 7$. Consider the induced homomorphisms

$$
\bar{h}_{*}: \pi_{r}\left(\Lambda^{\prime}\right) \rightarrow \pi_{r}(\Lambda), \quad h_{*}: \pi_{r+1}\left(\hat{A}^{\prime}\right) \rightarrow \pi_{r+1}(X),
$$

which are related by (7.3). We have

$$
\psi(\xi)=h_{*} E^{\prime}\left(\alpha^{\prime}\right), \quad \psi(\eta)=h_{*} E^{\prime}\left(\beta^{\prime}\right),
$$

by the definition of $h$. By (8.2a) and (7.3), however

$$
h_{*} E^{\prime}=h_{*} \psi^{\prime} j^{\prime}=\psi \bar{h}_{*} j^{\prime},
$$

and so it follows from (7.2) and (9.6) that

$$
\xi=\bar{h}_{*} j^{\prime}\left(\alpha^{\prime}\right), \quad \eta=\bar{h}_{*} j^{\prime}\left(\beta^{\prime}\right) .
$$

Therefore

$$
\begin{aligned}
\langle\xi, \eta\rangle & =\left\langle\bar{h}_{*} j^{\prime}\left(\alpha^{\prime}\right), \bar{h}_{*} j^{\prime}\left(\beta^{\prime}\right)\right\rangle \\
& =\bar{h}_{*}\left\langle j^{\prime}\left(\alpha^{\prime}\right), j^{\prime}\left(\beta^{\prime}\right)\right\rangle,
\end{aligned}
$$

by (2.2), since $\bar{h}$ is multiplicative. Since $\psi \bar{h}_{*}=h_{*} \psi^{\prime}$, by (7.3), it follows that

$$
\begin{aligned}
\psi\langle\xi, \eta\rangle & =h_{*} \psi^{\prime}\left\langle j^{\prime}\left(\alpha^{\prime}\right), j^{\prime}\left(\beta^{\prime}\right)\right\rangle, \\
& =(-1)^{p} h_{*}\left[E^{\prime}\left(\alpha^{\prime}\right), E^{\prime}\left(\beta^{\prime}\right)\right], \text { by }(9.5), \\
& =(-1)^{p}\left[h_{*} E^{\prime}\left(\alpha^{\prime}\right), h_{*} E^{\prime}\left(\beta^{\prime}\right)\right], \text { by naturality, } \\
& =(-1)^{p}[\psi(\xi), \psi(\eta)], \text { by }(9.6) .
\end{aligned}
$$

This proves (8.7), and completes the proof of the various other theorems which we have deduced from it. 


\section{APPENDIX}

10. Separation elements. The notion of a separation element is not exactly a special case of the notion of a separation cochain (see [1]). Hence we provide a brief account in this Appendix.

Let $S^{r}(r \geq 1)$ denote the unit sphere in euclidean $(r+1)$-space, and let $S^{r-1}$ denote its equator. Let $V^{r}$ denote the convex hull of the equator, and let $E_{+}, E_{-}$denote the two hemispheres into which $S^{r-1}$ divides $S^{r}$. Let $p, q: V^{r} \rightarrow S^{r}$ denote the orthogonal projections of $V^{r}$ onto $E_{+}, E_{-}$, respectively, (orthogonal to the plane of $V^{r}$ ).

Let $K$ be a $C W$-complex with a subcomplex $L$ such that $K-L=e^{r}$, an open $r$-cell. That is to say, $e^{r}$ is the topological image of the interior of $V^{r}$ under a map $f: V^{r} \rightarrow K$ such that $f S^{r-1} \subset L$. Let $u, v: K \rightarrow X$ be maps which agree on $L$, where $X$ is a space. Then we define a map $g: S^{r} \rightarrow X$ by

$$
g p=u f, \quad g q=v f .
$$

We define $d(u, v)$, the separation element of $u$ and $v$, to be the homotopy class of $g$ in $\pi_{r}(X)$. The following relations are easily verified.

THEOREM (10.2). Let $u, v: K \rightarrow X$ be maps which agree on $L$. Then $u \cong v$, relative to $L$, if, and only if, $d(u, v)=0$.

Corollary (10.3). If $u: K \rightarrow X$ is a map then $d(u, u)=0$.

THEOREM (10.4). Let $u, v, w: K \rightarrow X$ be maps which agree on $L$. Then $d(u, w)=d(u, v)+d(v, w)$.

Corollary (10.5). Let $u, v: K \rightarrow X$ be maps which agree on $L$. Then $d(u, v)+d(v, u)=0$.

THEOREM (10.6). Let an element $\delta \in \pi_{r}(X)$ and a map $u: K \rightarrow X$ be given. Then there exists a map $v: K \rightarrow X$ which agrees with $u$ on $L$ such that $d(u, v)=\delta$.

Theorem (10.7). Let $u_{t}, v_{t}: K \rightarrow X$ be homotopies which agree on $L$, where $0 \leqq t \leqq 1$. Then $d\left(u_{0}, v_{0}\right)=d\left(u_{1}, v_{1}\right)$.

Theorem (10.8). Let $u, v: K \rightarrow X$ be maps which agree on $L$. Let $h: X \rightarrow Y$ be a map, where $Y$ is a space. Then $d(h u, h v)$ is equal to the image of $d(u, v)$ under the homomorphism induced by $h$. 
Theorem (10.9). Let $k:(K, L) \rightarrow\left(K^{\prime}, L^{\prime}\right)$ be a map of degree $p$, where $K$ and $K^{\prime}$ are $C W$-complexes with subcomplexes $L$ and $L^{\prime}$, respectively, which are complements of r-cells in their respective complexes. Let $u, v: K^{\prime} \rightarrow X$ be maps which agree on $L^{\prime}$. Then $d(u k, v k)=p d(u, v)$.

\section{REFERENCES}

1. S. Eilenberg, Cohomology and continuous mappings, Ann. of Math., 41 (1940), 231-251.

2. I. M. James, Reduced product spaces, Ann. of Math., 62 (1955), 170-197.

3. I. M. James, On the suspension triad, Ann. of Math., 63 (1956), 191-247.

4. J. C. Moore, The double suspension ..., Bol. Soc. Mat. Mexicana (2) 1 (1956), 28-37.

5. N. E. Steenrod, The topology of fibre bundles, Princeton University Press, 1951.

6. G. W. Whitehead, On mappings into group-likc spaces, Comm. Math. Helv., 28 (1954), 320-328.

The Institute for Advanced Study 


\section{PACIFIC JOURNAL OF MATHEMATICS}

\section{EDITORS}

H. L. ROYDEN

Stanford University

Stanford, California

R. A. Beaumont

University of Washington

Seattle 5, Washington

\section{A. L. Whiteman}

University of Southern California

Los Angeles 7, California

E. G. Straus

University of California

Los Angeles 24, California

\section{ASSOCIATE EDITORS}
E. F. BECKENBACH
C. E. BURGESS
M. HALL
E. HEWITT

\author{
A. HORN \\ V. GANAPATHY IYER \\ R. D. JAMES \\ M. S. KNEBELMAN
}

L. NACHBIN

I. NIVEN

G. SZEKERES

T. G. OSTROM

M. M. SCHIFFER
F. WOLF

K. YOSIDA

\section{SUPPORTING INSTITUTIONS}

\author{
UNIVERSITY OF BRITISH COLUMBIA \\ CALIFORNIA INSTITUTE OF TECHNOLOGY \\ UNIVERSITY OF CALIFORNIA \\ MONTANA STATE UNIVERSITY \\ UNIVERSITY OF NEVADA \\ OREGON STATE COLLEGE \\ UNIVERSITY OF OREGON \\ UNIVERSITY OF SOUTHERN CALIFORNIA
}

\author{
STANFORD UNIVERSITY \\ UNIVERSITY OF UTAH \\ WASHINGTON STATE COLLEGE \\ UNIVERSITY OF WASHINGTON \\ AMERICAN MATHEMATICAL SOCIETY \\ CALIFORNIA RESEARCH CORPORATION \\ HUGHES AIRCRAFT COMPANY \\ THE RAMO-WOOLDRIDGE CORPORATION
}

Mathematical papers intended for publication in the Pacific Journal of Mathematics should be typewritten (double spaced), and the author should keep a complete copy. Manuscripts may be sent to any of the editors. All other communications to the editors should be addressed to the managing editor, E. G. Straus at the University of California, Los Angeles 24, California.

50 reprints per author of each article are furnished free of charge; additional copies may be obtained at cost in multiples of 50 .

The Pacific Journal, of Mathematics is published quarterly, in March, June, September, and December. The price per volume (4 numbers) is $\$ 12.00$; single issues, $\$ 3.50$. Back numbers are available. Special price to individual faculty members of supporting institutions and to individual members of the American Mathematical Society: $\$ 4.00$ per volume; single issues, $\$ 1.25$.

Subscriptions, orders for back numbers, and changes of address should be sent to Pacific Journal of Mathematics, 2120 Oxford Street, Berkeley 4, California.

Printed at Kokusai Bunken Insatsusha (International Academic Printing Co., Ltd.), No. 10, 1-chome, Fujimi-cho, Chiyoda-ku, Tokyo, Japan.

PUBLISHED BY PACIFIC JOURNAL OF MATHEMATICS, A NON-PROFIT CORPORATION

The Supporting Institutions listed above contribute to the cost of publication of this Journal, but they are not owners or publishers and have no responsibility for its content or policies. 


\section{Pacific Journal of Mathematics}

\section{Vol. 7, No. 2 \\ February, 1957}

William F. Donoghue, Jr., The lattice of invariant subspaces of a completely continuous quasi-nilpotent transformation ................... 1031

Michael (Mihály) Fekete and J. L. Walsh, Asymptotic behavior of restricted extremal polynomials and of their zeros.................... 1037

Shaul Foguel, Biorthogonal systems in Banach spaces ............... 1065

David Gale, A theorem on flows in networks ................... 1073

Ioan M. James, On spaces with a multiplication .................. 1083

Richard Vincent Kadison and Isadore Manual Singer, Three test problems in operator theory .................................... 1101

Maurice Kennedy, A convergence theorem for a certain class of Markoff processes........................................ 1107

G. Kurepa, On a new reciprocity, distribution and duality law ........ 1125

Richard Kenneth Lashof, Lie algebras of locally compact groups ........ 1145

Calvin T. Long, Note on normal numbers .................... 1163

M. Mikolás, On certain sums generating the Dedekind sums and their reciprocity laws ..................................... 1167

Barrett O'Neill, Induced homology homomorphisms for set-valued maps......................................... 1179

Mary Ellen Rudin, A topological characterization of sets of real numbers........................................... 1185

M. Schiffer, The Fredholm eigen values of plane domains 1187

F. A. Valentine, A three point convexity property .........

Alexander Doniphan Wallace, The center of a compact lattice is totally

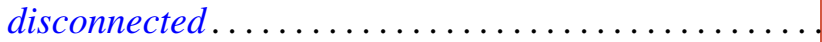

Alexander Doniphan Wallace, Two theorems on topological lattices.

G. T. Whyburn, Dimension and non-density preservation of mappings...

John Hunter Williamson, On the functional representation of certain algebraic systems ... 